\title{
REVIEW ARTICLE Imprinting of the immune system by the microbiota early in life
}

\author{
Ziad Al Nabhani ${ }^{1}$ and Gérard Eberl ${ }^{1}$
}

The ontogeny and maturation of the immune system is modulated by the microbiota. During fetal life, the mother's microbiota produces compounds that are transferred to the fetus and offspring, and enhance the generation of innate immune cells. After birth, the colonizing microbiota induces the development of intestinal lymphoid tissues and maturation of myeloid and lymphoid cells, and imprints the immune system with a reactivity level that persists long after weaning into adulthood. When the cross-talk between host and microbiota is perturbed early in life, a pathological imprinting may develop that is characterized by excessive immune reactivity in adulthood, which translates into increased susceptibility to inflammatory pathologies. In this review, we discuss the recent data that demonstrate the existence of a time window of opportunity early in life during which mice and human have to be exposed to microbiota in order to develop a balanced immune system. We also discuss the factors involved in imprinting, such as the microbiota, immune cells and stromal cells, as well as the nature of imprinting.

Mucosal Immunology (2020) 13:183-189; https://doi.org/10.1038/s41385-020-0257-y

\section{INTRODUCTION: MICROBES, HYGIENE AND IMPRINTING OF THE IMMUNE SYSTEM}

The way we grow up and learn to interact with others affects us lifelong. Several of our physiological systems, such as the nervous and immune systems, directly sense the environment and respond to it in order to maintain homeostasis and survival of the individual. From birth on, the individual is exposed to new types of cells, the microbes, which activate the immune system and induce the development and maturation of immune cells and organs that contribute to the development of a functional symbiosis between host and microbiota., ${ }^{1,2}$ In both human and mice, a paucity of bacteria early in life leads to immunological defects that can be sustained into adulthood and increase the susceptibility to develop chronic inflammatory pathologies. 3,4

The hygiene hypothesis

A decreased incidence in infectious diseases during the $20^{\text {th }}$ century is associated with an increase in inflammatory pathologies, such as allergy and autoimmunity, which inspired the formulation of the hygiene hypothesis. ${ }^{5}$ This hypothesis, or a generalized version of it, states that decreased exposure to microbes leads to deregulation of the immune system and a consequent increase in pathogenic reactivity. It is supported both by epidemiological observations in human and experiments in mice. For example, children raised in the proximity of farm animals, or given non-sterilized pacifiers, develop significantly less allergy than children raised away from animals or excessively protected from microbes. ${ }^{6,7}$ In mice, the absence of microbes in germ-free (GF) animals, or the presence of a low diversity microbiota, is associated with increased levels of proallergic cells, cytokines and $\lg \mathrm{E}^{8,9}$
A time window of opportunity

Adding to the increased susceptibility to immunopathology associated with excessive hygiene is a temporal window during which exposure to microbes appears critical for the development of a balanced immune system (Fig. 1). A growing literature demonstrates that bacteria and their products modulate the development of the immune system during the period spanning birth to weaning (the neonatal period), with long term consequences on immune reactivity and immunopathology. ${ }^{4,10}$ This period is therefore termed a "window of opportunity" that allows for an important host-microbe cross-talk to occur and that cannot be missed. For instance, mice treated with antibiotics or inhibitors of antigen transfer (through the intestinal barrier) before weaning, but not after, and GF mice colonized with microbiota after weaning but not before, develop increased susceptibility to allergy and IBD in adulthood. ${ }^{11-15}$ While earlier studies have focused on the intestine, similar observations were reported for the lung and skin, thus generalizing the "window of opportunity" concept to other mucosal surfaces. ${ }^{16-19}$ A similar "window of opportunity" may exist during late fetal life, ${ }^{10,20,21}$ even though its consequences, mechanisms and time limits remain to be more precisely defined.

Imprinting of the immune system

These observations have crystalized into the notion of "early life imprinting" of the immune system, a form of memory that translates the perinatal microbe-host crosstalk into long term setting of immune reactivity. ${ }^{15}$ Pathological imprinting, in particular, is defined as the increased susceptibility to inflammatory pathology developed by individuals that have not been exposed to microbiota, or possibly to a dysbiotic microbiota, during the window of opportunity. Several studies are now

\footnotetext{
${ }^{1}$ Microenvironment and Immunity Unit, Institut Pasteur, INSERM U1224, Paris, France
}

Correspondence: Gérard Eberl (gerard.eberl@pasteur.fr)

Received: 23 November 2019 Revised: 7 January 2020 Accepted: 8 January 2020

Published online: 27 January 2020 
Pathological imprinting

Absent/dysbiotic microbiota

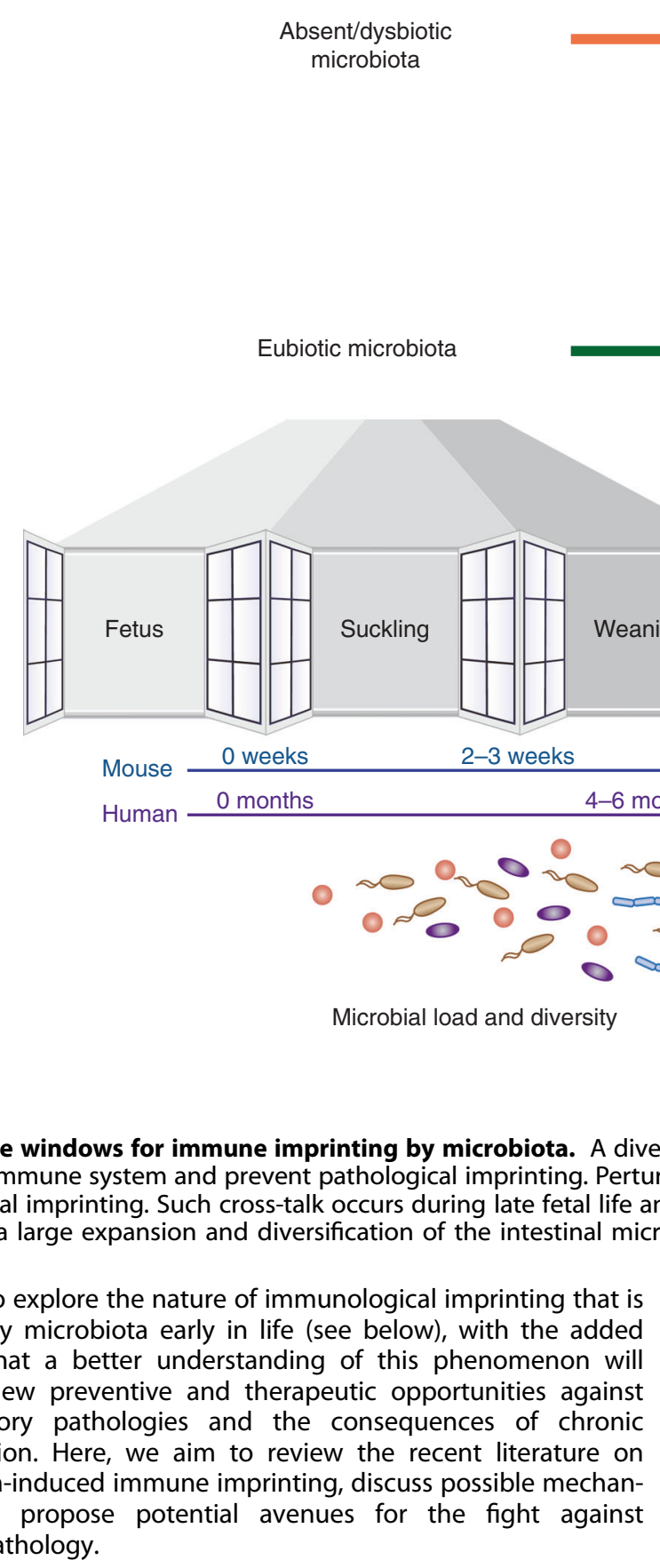

Fig. 1 Time windows for immune imprinting by microbiota. A diverse microbiota in the mother and the offspring is required to develop a balanced immune system and prevent pathological imprinting. Perturbation of the microbiota alters the host-microbe cross-talk and leads to pathological imprinting. Such cross-talk occurs during late fetal life and during the neonatal period spanning birth to weaning, a period that witnesses a large expansion and diversification of the intestinal microbiota.

devoted to explore the nature of immunological imprinting that is induced by microbiota early in life (see below), with the added wisdom that a better understanding of this phenomenon will uncover new preventive and therapeutic opportunities against inflammatory pathologies and the consequences of chronic inflammation. Here, we aim to review the recent literature on microbiota-induced immune imprinting, discuss possible mechanisms, and propose potential avenues for the fight against immunopathology.

\section{TIMES WINDOWS OF OPPORTUNITY}

Stable imprinting of the immune system by microbiota and its products has been reported to occur during different periods of time before and after birth.

\section{A fetal time window?}

Numerous experiments and epidemiologic data report an effect of the maternal microbiota, or of antibiotic treatment of the pregnant mother, on the immune system and susceptibility to inflammatory pathology of the offspring. ${ }^{10,20-26}$ During pregnancy, controversial evidence has suggested the existence of bacteria residing within the placenta, which could have an impact on the fetal immune system. ${ }^{27,28}$ More likely, the microbiota of the pregnant mother produces compounds that affect the immune system of the fetus or the newborn (Fig. 2). Short chain fatty acids (SCFA) produced by bacteria through fermentation of dietary fibers, such as acetate, imprint the fetal lung with an increased potential to induce the generation of regulatory T cells (Tregs) in adult offsprings and reduce the severity of house dust mite (HDM)-induced allergy. ${ }^{10}$ This in line with a demonstrated effect of SCFA on the generation of Tregs via the G-coupled protein receptors Gpr41 and Gpr43, as well as through direct inhibition of histone deacetylases (HDAC). ${ }^{29,30}$

Bacterial ligands of the aryl hydrocarbon receptor (Ahr) have also been shown to affect fetal or neonatal immunity. Using an auxotrophic $E$. coli strain that was present only in the pregnant mother, it was demonstrated that Ahr ligands produced by $E$. coli were transferred from the mother to the fetus and newborn via an antibody-dependent transport mechanism. ${ }^{20}$ In the offspring, these ligands boosted the number and activity of myeloid cells, as well as of type 3 innate lymphoid cells (ILC3), an effect reported earlier by different teams. ${ }^{31-33}$ However, these studies did not show immune imprinting of the offspring, but rather, that perturbations of the maternal microbiota during pregnancy can impact the offpring and alter the development of its immune 


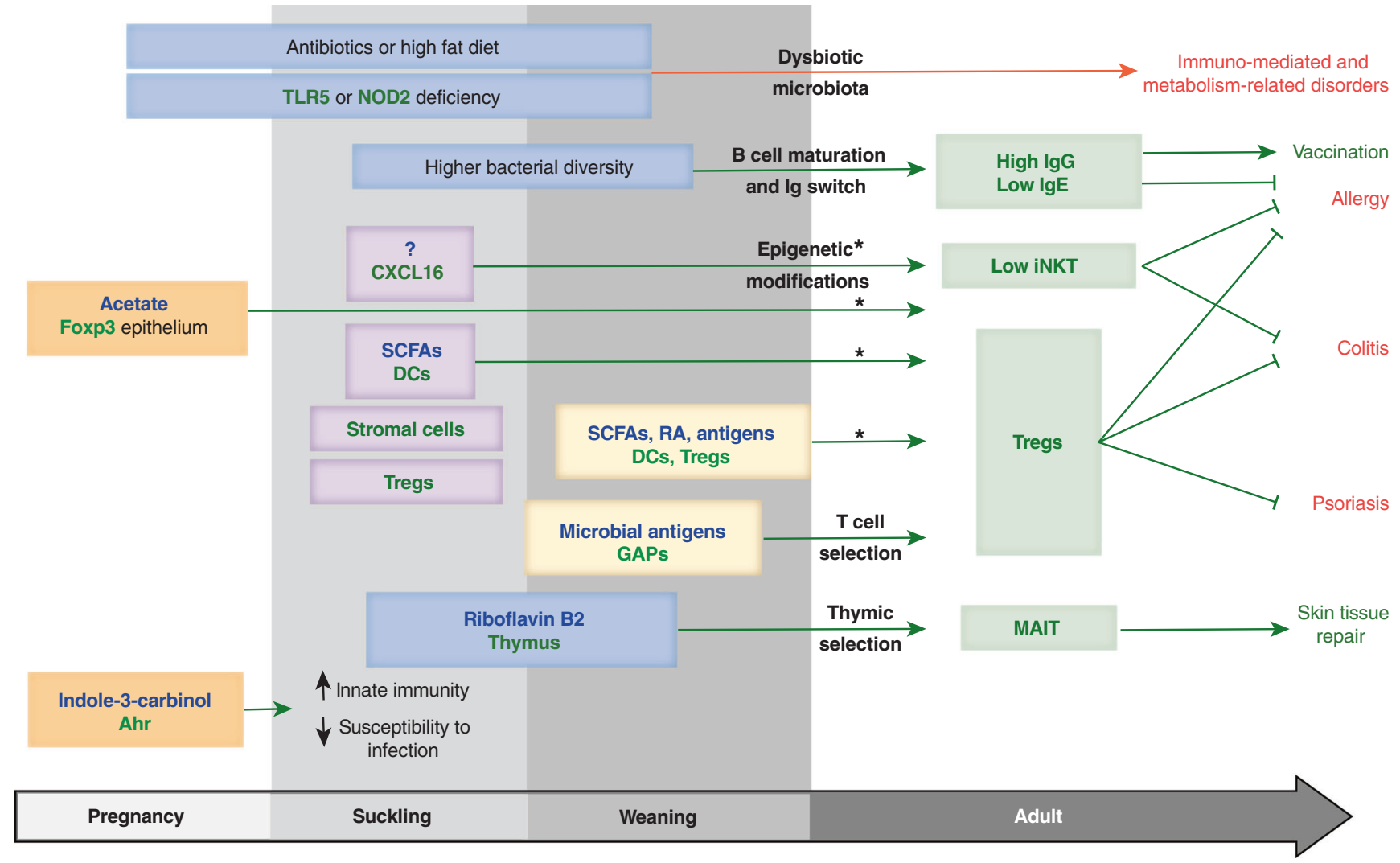

Fig. 2 Dietary, bacterial and host elements involved in immune imprinting. Compounds of dietary and/or bacterial origin (blue text) affect host genes, pathways and cells (green) during different periods of time before and after birth, and thereby impact the development and imprinting of the immune system, as well as the composition of the microbiota. The reactivity of the immune system later in life will determine the susceptibility of the host to inflammatory pathologies and the consequences of chronic inflammation, as well as its reactivity to immunotherapy, vaccination and tissue repair. On the right, positive effects are shown by green arrows and text, whereas negative effects are shown by red arrows and text. *Epigenetic modifications. Ahr, aryl hydrocarbon receptor; CXCL16, C-X-C Motif Chemokine Ligand 16; DCs, dendritic cells; Foxp3, forkhead box P3; GAPs, Goblet cells associated antigen passages; Ig, immunoglobulin; iNKT, invariant natural killer T cells; MAIT, mucosal associated invariant T cells; RA, retinoic acid; SCFAs, short chain fatty acids; Tregs, regulatory T cells.

system. Similarly, alterations (dysbiosis) in the maternal microbiota can be transmitted to the offspring and determine immune reactivity in the long term, ${ }^{34-36}$ an effect that we will discuss in a later chapter.

Other compounds of dietary origin, the metabolism of which may be modulated by the microbiota, such as retinoic acid (RA), have significant effects on the developing fetal immune system. ${ }^{37}$ However, these effects are of an ontogenic nature that will not be discussed here.

The neonatal time window

Imprinting of the immune system by the microbiota has been best demonstrated during the neonatal period. ${ }^{4}$ The colonizing microbiota has a profound impact on the development of the intestinal immune system. For example, GF mice fail to develop isolated lymphoid follicles (ILFs) during the second week after birth, 2,38 recruit low numbers of intra-epithelial lymphocytes $(\mathrm{IEL}){ }^{39}$ fail to generate RORyt ${ }^{+}$Tregs $^{40,41}$ and show a decrease in the substitution of yolk-sac-derived macrophages with bone marrow-derived macrophages. ${ }^{42}$ However, these effects are mostly transient and can be salvaged, as far as we know, by microbial colonization later in life. However, other effects of the microbiota during the neonatal period are long-lived and apparently irreversible. Nagler's team has pioneered experimental demonstration that early life treatment of mice with antibiotics has an effect into adulthood, long after cessation of treatment, and manifested by an increased susceptibility to allergy. ${ }^{11}$ This phenomenon was confirmed later by Finlay's and Blumberg's teams. ${ }^{12,13}$ They showed that mice treated with antibiotics during the neonatal period, but not after, or colonization of GF mice only after weaning, developed increased susceptibility to allergy in gut and lung. Exposure to microbiota during the neonatal period also diminished the susceptibility to colitis-associated tumorigenesis. ${ }^{15,43}$ Similarly, treatment of mice with antibiotics until weaning led to increased susceptibility to skin and lung fibrosis in a model of systemic sclerosis. ${ }^{18}$ Furthermore, using the house dust mite (HDM) model of lung allergy, Marsland's team showed that exposure of mice to microbiota during the second week after birth imprinted efficient generation of Tregs later in life, which modulated HDM-induced inflammation. ${ }^{16}$ A similar observation by Rosenblum's team was reported for the generation of skin-resident Tregs that regulate microbiota-induced inflammation in adulthood. ${ }^{17}$ Finally, in a process affecting all mucosal surfaces, it was shown that bacterial metabolites of vitamin B2 are required before weaning to induce the generation of mucosa-associated invariant T (MAIT) cells in the adult. ${ }^{44,45}$

\section{The weaning reaction}

Another twist in the story was added by the characterization of the weaning reaction. We reported that the weaning reaction is the first vigorous immune response to the colonizing intestinal microbiota after birth. ${ }^{15}$ As mice diversify their food intake from a milk-only diet to one that includes solid food, during the third week after birth, the intestinal microbiota expands dramatically both in numbers of bacteria and richness of bacterial species. The host responds by developing the weaning reaction, characterized 
by high levels of pro-inflammatory cytokines in the intestine, such as IFN $Y$ and TNFa, which recedes during the fourth week after birth. Remarkably, such a vigorous reaction is not elicited by the colonization by microbiota of adult GF mice, demonstrating the existence of a restricted time window of opportunity for the weaning reaction. When this reaction was inhibited by transient antibiotic treatment, ${ }^{15}$ or perturbed by excessive calorie intake, ${ }^{46}$ mice developed increased susceptibility to inflammatory pathology in adulthood, which was characterized by broad exacerbation of cytokine production upon challenge. Similar observations were reported in transgenic mice expressing a T-cell receptor specific for bacterial flagellin. ${ }^{14}$ Using this model, Newberry's team showed that the time window opens during the second week after birth as Goblet cells increase their capacity to transfer antigens from the intestinal lumen to the lamina propria, in a process termed Goblet cell-associated antigen passage (GAP). ${ }^{47}$ While declining levels of epidermal growth factor (EGF) in the mother's milk allow for the opening of the time window, it remains unclear how the time window closes after weaning, although detection of microbes by Myd88-dependent innate receptors may be involved.

Finally, even though antibiotic treatments after weaning affects the microbiota, and therefore, the activation state of the immune system and immune adaptive memory, microbial exposure after weaning did not alter pathological imprinting. ${ }^{15}$ Therefore, epidemiological data reporting effects of antibiotic treatment during childhood on susceptibility to allergy may not be a consequence of immune imprinting, but rather, of dysbiosis and the consequent altered activation state of the immune system (discussed below).

\section{MECHANISMS OF IMPRINTING}

Arguably, imprinting is another word for memory. For an immunologist, however, memory is associated with antigen specificity carried by B and T cells, while other forms of not so specific memory have been diversely termed innate memory, adaptation, training or imprinting. ${ }^{48-51}$ Historically, Konrad Lorenz used the term imprinting to describe how early experience defines animal behavior later in life. ${ }^{52} \mathrm{He}$ went on to state that imprinting may only occur during "a narrowly defined period in the individual's life" and that the imprinted behavior "cannot be forgotten". Therefore, imprinting fits quite well with the immunological phenomenon we are discussing here.

More interesting than semantics are probably the mechanisms involved in encoding memory. If memory is encoded as a durable change of state in a system, then it can take many forms. Antigenspecific memory is encoded by a genetic re-arrangement of gene segments coding for antigen receptors in lymphocytes, followed by selection and amplification of cell clones. ${ }^{53}$ Of note, amplification of cell clones or distinct cell populations is a common mode of memory in both adaptive and innate immunity, as well as in the selection of stem cells, cancer cells, and microbiota. ${ }^{49-51}$ Other forms of memory are encoded in the nervous system, such as circuit structure and synaptic strength. ${ }^{54}$ Finally, the epigenome is a particularly attractive substrate of memory, as it can be modified and maintained over several cell divisions, and transmitted from one cell to another (as in the case of sperm cells). ${ }^{55}$ Epigenetic memory is also interesting, as it is induced by experience rather than by chance (as in the case of antigen receptor genes), and propagates an adaptation to the environment-a Lamarckian principle of evolution.

The colonization of the intestine by microbiota during the neonatal period induces significant transcriptional changes in the adult intestine as compared to GF mice colonized as adults, which encompass many different systems. ${ }^{56}$ We therefore expect that diverse types of cells, tissues and organs are involved in immunological imprinting, using diverse modes of memory encoding.
Imprinting of lymphoid and myeloid cells

The model of oxazolone-induced colitis is driven by the recruitment to the intestine of invariant NKT (iNKT) cells that produce IL-13. The colonizing microbiota alters the methylation pattern of the gene coding for CXCL16, the chemokine that recruits NKT cells to the intestine. ${ }^{13}$ Such microbiota-induced epigenetic modifications must occur before weaning to decrease iNKT recruitment, possibly involving CD1d-mediated antigen presentation to iNKT cells during the weaning reaction. ${ }^{57}$ While the probable carrier of imprinting is dendritic cells (DC), it remains unclear how such epigenetic changes are induced.

We have shown that microbiota-induced RORyt ${ }^{+}$Tregs must be generated during the weaning reaction in order to prevent pathological imprinting. ${ }^{15}$ All components required to induce $\mathrm{RORYt}^{+}$Tregs, i.e., pro-inflammatory bacteria and bacterial antigens, SCFA and RA, must therefore be present during weaning. In preliminary experiments, we show that RORyt ${ }^{+}$Tregs carry protective imprinting, but the nature of imprinting remains to be identified. Intriguingly, during fetal life, SCFA were reported to induce the imprinting of Treg responses later in life, but lung epithelial cells, and not Tregs, were the carrier of imprinting. ${ }^{10}$ SCFA increased acetylation of the gene coding for FoxP3, a master regulator of Treg generation, through inhibition of HDAC9. In this case, the mechanism by which microbiota modifies the epigenome is rather clear, but the link between epithelial and $T$ cell expression of FoxP3 remains to be understood.

Antigen-specific memory can carry imprinting from the neonatal period into adulthood. ${ }^{14}$ In mice transgenic for a T cell receptor recognizing Lachnospiracea flagellin, the generation of $\mathrm{RORYt}^{+}$Tregs occurred mainly during the weaning reaction and protected from severe colitis later in life. The restrictive period of Treg generation in this context is surprising, as microbiota can induce RORyt ${ }^{+}$Tregs in the adult. ${ }^{40}$ Protection from colitis is expected to be antigen-specific, and therefore, may not apply to protective imprinting in mice carrying complex microbiotas and $\mathrm{T}$ cell repertoires. A similar phenomenon was observed in the skin. Using mice transgenic for a $\mathrm{T}$ cell receptor recognizing a Staphylococcus epidermidis antigen, it was shown that antigenspecific Tregs must be generated during the second week after birth in order to maintain tolerance to this antigen in the adult. ${ }^{17}$

Protective imprinting in the lung requires exposure to microbiota during the second week after birth. ${ }^{16}$ The protection is mediated by Tregs, but early life exposure involves the expansion of dendritic cells (DC) expressing PD-L1 that are required to induce the expansion of Tregs later in life, suggesting that DCs carry imprinting. In the case of MAIT cells, Belkaid's team showed that, for their generation, riboflavin metabolites must be produced by the microbiota before weaning. ${ }^{44}$ Together with Lantz's demonstration that these metabolites are required for the thymic generation of MAIT cells, ${ }^{45}$ the data suggest that the presentation of vitamin B2 metabolites by double positive thymocytes is critical early in life to maintain the pool of MAIT cells in a lifetime.

However, T cells can also carry pathological imprinting, as reported in the context of IBD. ${ }^{58} \mathrm{CD} 4^{+} \mathrm{T}$ cells isolated from mice that were treated with antibiotics during the neonatal period induced a more severe colitis than cells isolated from untreated mice when transferred into RAG-deficient mice. It was suggested that $\mathrm{CD} 4^{+} \mathrm{T}$ cells carry pathological imprinting through dysregulated expression of genes associated with cell cycle, metabolism and stress.

Finally, B cells may carry imprinting. McCoy's team showed that high levels of serum IgE in GF mice were normalized only in mice exposed to complex microbiota before 4 weeks of age. ${ }^{9}$ Furthermore, antibiotic treatment during the neonatal period lead to decreased IgG responses to various vaccines in the adult, while IFNy production by T cells was increased. ${ }^{59}$ However, as $T$ helper cells are involved in B cell activation and isotype switching, imprinting in $T$ cells cannot be ruled out in these cases. 
Imprinting of bone marrow cells

The imprinting of progenitors of lymphoid and myeloid cells in the bone marrow seems plausible, as microbial products have been shown to alter hematopoiesis. Furthermore, imprinting of bone marrow progenitors may be stable over time and affect the immune system in most tissues. Bacterial compound and metabolites, such as peptidoglycan and SCFA, increase myelopoiesis and the production of neutrophils, macrophages and DCs in neonates and adults, ${ }^{60-63}$ while GF mice show reduced hematopoiesis. ${ }^{64}$ The effect of SCFA on the generation of macrophages and DCs is dependent on Gpr41 and Gpr43, and may therefore involve epigenetic reprogramming of their progenitors. ${ }^{62}$ Possibly a consequence of similar mechanisms, adult rats that were raised by mothers fed on a protein-poor diet develop reduced neutrophil activity, indicating early life imprinting of myeloid progenitors. ${ }^{65}$ Evidence for similar effects on lymphoid progenitors remains scarce, even though endotoxins, such as lipopolysaccharides (LPS), affect $B$ cell maturation. ${ }^{66}$

Imprinting of epithelial cells

Exposure to microbial compounds early in life regulates the ability of epithelial cells to activate innate immune pathways. Upon bacterial colonization of the gut or exposure to allergen in the lung, Toll-like receptor (TLR)-mediated signaling in epithelial cells is downregulated through negative control loops provided by microRNA-146a and A20. ${ }^{67-69}$ We therefore expect that microbiota-induced regulation of epithelial function affects the development of the immune system and imprinting. However, in the intestine, the epithelium is entirely renewed approximately every 5 days, suggesting that the more likely carriers of imprinting are epithelial stem cells. A precedent for imprinting of epithelial stem cells has been recently reported for the skin. An initial wound healing leads to inflammation-induced epigenetic modifications in stem cells, which endows them with increased reactivity to subsequent challenges. ${ }^{70}$ Whether neonatal exposure to the colonizing microbiota leads to a similar epigenetic imprinting in intestinal epithelial stem cells is an interesting and consequence-rich possibility.

Imprinting of stromal cells

Imprinting of stromal cells by microbiota has been demonstrated in the gut-draining mesenteric lymph nodes $(\mathrm{mLN})$. Stromal (fibroblastoid $\mathrm{CD} 31^{-} \mathrm{gp}^{+} 8^{+}$) cells in $\mathrm{mLN}$ promote the generation of Tregs, a property that could be maintained in the long term upon transfer into other lymph node sites. ${ }^{71}$ Also maintained was their distinct expression pattern, including increased expression of Retinaldehyde dehydrogenase (Raldh2/3) that converts vitamin A into RA, an efficient promoter of Tregs. ${ }^{72}$ While imprinting of $\mathrm{mLN}$ could occur early in life, it was shown that imprinting could also be induced in the adult. ${ }^{71}$ The nature of imprinting, probably involving epigenetic modifications, remains to be determined.

Imprinting of microbiota

A large body of evidence shows that perturbation, or dysbiosis, of the microbiota early in life can persist into the long term and increase susceptibility to inflammatory pathology in the adult ${ }^{3}$ or alter the response to vaccination. ${ }^{59}$ Dysbiosis can have multiple sources. The first is the mother. Dybiosis of the maternal microbiota, induced by antibiotics, food or inflammation, can be transmitted to the offsprings, which then carry it into adulthood. ${ }^{10,22-26}$ The second is direct perturbation of the neonate's microbiota by antibiotics, which modify the ecological equilibrium in the intestine (and elsewhere). ${ }^{73}$ The mechanisms of irreversible perturbations of the microbiota by antibiotics have been nicely shown in the context of vancomycin treatment in patients and mice, which creates the opportunity for vancomycinresistant Enterococci to dominate the microbiota. ${ }^{74}$ Food is another major modifier of microbiota. In malnourished children, a persistent immature microbiota prevents efficient refeeding and normal growth of these children. ${ }^{75}$ In mice, we have reported that excessive calorie intake during the neonatal period leads to the expansion of sulfide-producing bacteria that destabilize the mucus and thereby induce pathological imprinting. ${ }^{46}$ Nevertheless, we did not observe long term imprinting of the microbiota in this case. Finally, the genetic background of the host has important consequences on the composition of the neonatal microbiota that is maintained into adulthood and may shape susceptibility to inflammatory pathology. Mice deficient in TLR5 lose early the ability to control flagellated bacteria, with long term consequences on the microbiota composition, ${ }^{76}$ and mice deficient in NOD2 develop a microbiota different from wild type mice when treated with antibiotics during the neonatal period, with consequences on susceptibility to colitis in the adult. ${ }^{77}$

How dysbiosis increases susceptibility to colitis and allergy in the adult remains to be clearly understood. During perturbation of the microbiota, bacteria or fungi (or viruses) endowed with increased invasive potentials may induce chronic inflammation of the mucosal tissue, thereby increasing the risk of developing inflammatory pathology upon challenge. ${ }^{74}$ For example, arthritisprone $\mathrm{K} / \mathrm{BxN}$ mice develop the disease when mono-colonized with Segmented Filamentous Bacteria (SFB), as a consequence of the ability of SFB to induce type 3 immune responses characterized by the production of IL-23 and IL-17 (ref. ${ }^{78}$ ). A change in the microbial balance may also modify the equilibrium between different types of immune responses, and thereby increase the risk of inflammatory pathology driven by one type of immune response. ${ }^{79}$ This phenomenon of immune cross-regulation has been best demonstrated in the context of superinfections. For example, intestinal helminth infection negatively regulates the response and control of norovirus, ${ }^{80}$ while the elimination of a substantial fraction of the intestinal microbiota with antibiotics increases the response to norovirus by weakening such negative regulation. ${ }^{81}$

\section{Imprinting of the nervous system}

The nervous system is extremely sensitive to sensory input and imprinting during the neonatal period. ${ }^{82}$ For example, sounds have to be heard and spoken by the young child before the age of 1 year in order for the child to correctly pronounce them long after that period. ${ }^{83}$ Another spectacular example of neuronal imprinting is the sound of mother in hatching geese, which associate the first animal sound they hear to their mother, for life. ${ }^{84}$ It is therefore tempting to speculate that immune imprinting induced during the neonatal period may be carried, at least in part, by the nervous system. While the impact of the microbiota on brain function is now well supported, ${ }^{85}$ few studies yet document neonatal immune imprinting of the nervous system by the microbiota. In that line of ideas, it was demonstrated that exposure to microbiota during the neonatal period restores fear extinction learning in adulthood. ${ }^{86}$

\section{PREVENTING AND REVERSING PATHOLOGICAL IMPRINTING}

The lack of exposure, or exposure to a low diversity or dysbiotic microbiota, during the neonatal period leads to pathological imprinting and increased susceptibility to inflammatory pathologies later in life. Therefore, a sensible approach to prevent pathological imprinting is to identify components of the microbiota, as well as of the diet that nourishes both the host and the microbiota, which are required to ensure healthy imprinting. It is already established that particular bacterial strains promote healthy imprinting, such a fermenters of dietary fibers that generate SCFAs. ${ }^{15}$ Furthermore, the neonatal colonization of mice with Lactobacillus rhamnosus GG decreases susceptibility to colitis in the adult by inducing higher levels of $\operatorname{lgA} .^{87} \mathrm{RA}$, generated from dietary vitamin $A$, is required for a protective weaning reaction, ${ }^{15}$ 
and riboflavin metabolites are required during the neonatal period to generate MAIT cells $s^{44,45}$ Thus, a combination of bacteria and food supplement may be designed to favor healthy imprinting and decrease the incidence of inflammatory pathologies later in life.

In light of the current understanding of immunological imprinting, we may also avoid certain treatments and behaviors, whenever possible. For example, antibiotic treatment during the neonatal period, and exposure of weanlings to high fat diets are causally linked to pathological imprinting. However, antibiotic treatments save infants from infections, and high calorie food may be the only food available. In these cases, it would be desirable to identify markers in stool or blood during routine medical checks and envisage reversion of pathological imprinting if detected. If such pathological imprinting is characterized in the microbiota, dysbiosis may be corrected by dietary prebiotics or a new generation of probiotics. In blood, markers of pathological imprinting remain to be identified. Furthermore, the nature of pathological imprinting remains to be better understood in order to design methods to reverse it. Recent finding on the epigenetic nature of imprinting in myeloid ${ }^{13}$ and epithelial cells ${ }^{70}$ indicate that reversion of pathological imprinting will be challenging. Obviously, methods to revert pathological imprinting must be less pathogenic than pathological imprinting itself.

\section{CONCLUSION}

In conclusion, it is important to underscore that immune imprinting manifests itself through increased susceptibility to inflammatory challenges later in life. This increased susceptibility reflects higher reactivity of the immune system, which is a consequence of decreased immune-regulation or increased basal reactivity (probably the two faces of the same coin). A higher immune reactivity may appear pathogenic in the context of low pathogen pressure that is prevalent in societies with high hygiene and vaccination coverage. However, high immune reactivity may be beneficial in an environment that is contaminated with pathogens. Therefore, the notion of "pathological imprinting" may be contextual, as an individual with high immune reactivity may experience protective imprinting when infected, while an individual with low immune reactivity may appear pathologically imprinted in that context.

\section{ACKNOWLEDGEMENTS}

We thank all the members of the Microenvironment \& Immunity Unit, as well as from the Stroma, Inflammation \& Tissue Repair Unit, for support and discussion. Z.A.N. was supported by Pasteur-Roux Postdoctoral Fellowships from the Institut Pasteur. This work was supported by Institut Pasteur and INSERM, the Association François Aupetit, the Fondation pour la Recherche Medicale, Janssen Horizon, and an Innovator award from the Kenneth Rainin Foundation.

\section{AUTHOR CONTRIBUTIONS}

Z.A.N. and G.E. contributed equally to the manuscript.

\section{ADDITIONAL INFORMATION}

Competing interests: The authors declare no competing interests.

Publisher's note Springer Nature remains neutral with regard to jurisdictional claims in published maps and institutional affiliations.

\section{REFERENCES}

1. Koropatnick, T. A. et al. Microbial factor-mediated development in a hostbacterial mutualism. Science 306, 1186-1188 (2004)

2. Bouskra, D. et al. Lymphoid tissue genesis induced by commensals through NOD1 regulates intestinal homeostasis. Nature 456, 507-510 (2008).
3. Blaser, M. J. The theory of disappearing microbiota and the epidemics of chronic diseases. Nat. Rev. Immunol. 17, 461-463 (2017).

4. Gensollen, T., lyer, S. S., Kasper, D. L. \& Blumberg, R. S. How colonization by microbiota in early life shapes the immune system. Science 352, 539-544 (2016).

5. Bach, J. F. The effect of infections on susceptibility to autoimmune and allergic diseases. New Engl. J. Med 347, 911-920 (2002).

6. Riedler, J. et al. Exposure to farming in early life and development of asthma and allergy: a cross-sectional survey. Lancet 358, 1129-1133 (2001).

7. Hesselmar, B. et al. Pacifier cleaning practices and risk of allergy development. Pediatrics 131, e1829-e1837 (2013).

8. Kernbauer, E., Ding, Y. \& Cadwell, K. An enteric virus can replace the beneficial function of commensal bacteria. Nature 516, 94-98 (2014).

9. Cahenzli, J., Koller, Y., Wyss, M., Geuking, M. B. \& McCoy, K. D. Intestinal microbial diversity during early-life colonization shapes long-term IgE levels. Cell Host Microbe 14, 559-570 (2013).

10. Thorburn, A. N. et al. Evidence that asthma is a developmental origin disease influenced by maternal diet and bacterial metabolites. Nat. Commun. 6, 7320 (2015).

11. Bashir, M. E., Louie, S., Shi, H. N. \& Nagler-Anderson, C. Toll-like receptor 4 signaling by intestinal microbes influences susceptibility to food allergy. J. Immunol. 172, 6978-6987 (2004).

12. Russell, S. L. et al. Early life antibiotic-driven changes in microbiota enhance susceptibility to allergic asthma. EMBO Rep. 13, 440-447 (2012).

13. Olszak, T. et al. Microbial exposure during early life has persistent effects on natural killer T cell function. Science 336, 489-493 (2012).

14. Knoop, K. A. et al. Microbial antigen encounter during a preweaning interval is critical for tolerance to gut bacteria. Sci Immunol 2, eaoo1314 (2017).

15. Al Nabhani, Z. et al. A weaning reaction to microbiota is required for resistance to immunopathologies in the adult. Immunity 50, 1276-1288 (2019).

16. Gollwitzer, E. S. et al. Lung microbiota promotes tolerance to allergens in neonates via PD-L1. Nat. Med. 20, 642-647 (2014).

17. Scharschmidt, T. C. et al. A wave of regulatory T cells into neonatal skin mediates tolerance to commensal microbes. Immunity 43, 1011-1021 (2015).

18. Mehta, $\mathrm{H}$. et al. Early-life antibiotic exposure causes intestinal dysbiosis and exacerbates skin and lung pathology in experimental systemic sclerosis. J. Invest. Dermatol. 137, 2316-2325 (2017).

19. Zanvit, P. et al. Antibiotics in neonatal life increase murine susceptibility to experimental psoriasis. Nat. Commun. 6, 8424 (2015).

20. Gomez de Aguero, M. et al. The maternal microbiota drives early postnatal innate immune development. Science 351, 1296-1302 (2016).

21. Macpherson, A. J., de Aguero, M. G. \& Ganal-Vonarburg, S. C. How nutrition and the maternal microbiota shape the neonatal immune system. Nat. Rev. Immunol. 17, 508-517 (2017).

22. Nakajima, A. et al. Maternal high fiber diet during pregnancy and lactation influences regulatory $\mathrm{T}$ cell differentiation in offspring in mice. J. Immunol. 199, 3516-3524 (2017).

23. Metzler, S. et al. Association bet,en antibiotic treatment during pregnancy and infancy and the development of allergic diseases. Pediatr. Allergy Immunol. 30, 423-433 (2019).

24. Ortqvist, A. K., Lundholm, C., Halfvarson, J., Ludvigsson, J. F. \& Almqvist, C. Fetal and early life antibiotics exposure and very early onset inflammatory bowel disease: a population-based study. Gut 68, 218-225 (2019).

25. Munyaka, P. M., Eissa, N., Bernstein, C. N., Khafipour, E. \& Ghia, J. E. Antepartum antibiotic treatment increases offspring susceptibility to experimental colitis: a role of the gut microbiota. PLOS ONE 10, e0142536 (2015).

26. Miyoshi, J. et al. Peripartum antibiotics promote gut dysbiosis, loss of immune tolerance, and inflammatory bowel disease in genetically prone offspring. Cell Rep. 20, 491-504 (2017).

27. Aagaard, K. et al. The placenta harbors a unique microbiome. Sci. Transl. Med 6, 237ra265 (2014)

28. de Goffau, M. C. et al. Human placenta has no microbiome but can contain potential pathogens. Nature 572, 329-334 (2019).

29. Furusawa, Y. et al. Commensal microbe-derived butyrate induces the differentiation of colonic regulatory T cells. Nature 504, 446-450 (2013).

30. Smith, P. M. et al. The microbial metabolites, short-chain fatty acids, regulate colonic Treg cell homeostasis. Science 341, 569-573 (2013).

31. Kiss, E. A. et al. Natural aryl hydrocarbon receptor ligands control organogenesis of intestinal lymphoid follicles. Science 334, 1561-1565 (2011).

32. Lee, J. S. et al. AHR drives the development of gut ILC22 cells and postnatal lymphoid tissues via pathways dependent on and independent of Notch. Nat. Immunol. 13, 144-151 (2011).

33. Qiu, J. et al. The aryl hydrocarbon receptor regulates gut immunity through modulation of innate lymphoid cells. Immunity 36, 92-104 (2011).

34. Schulfer, A. F. et al. Intergenerational transfer of antibiotic-perturbed microbiota enhances colitis in susceptible mice. Nat. Microbiol. 3, 234-242 (2018). 
35. Ma, J. et al. High-fat maternal diet during pregnancy persistently alters the offspring microbiome in a primate model. Nat. Commun. 5, 3889 (2014).

36. Torres, J. et al. Infants born to mothers with IBD present with altered gut microbiome that transfers abnormalities of the adaptive immune system to germ-free mice. Gut 69, 42-51 (2020).

37. Larange, A. \& Cheroutre, H. Retinoic Acid and retinoic acid receptors as pleiotropic modulators of the immune system. Annu Rev. Immunol. 34, 369-394 (2016).

38. Hamada, $H$. et al. Identification of multiple isolated lymphoid follicles on the antimesenteric wall of the mouse small intestine. J. Immunol. 168, 57-64 (2002).

39. Takimoto, $\mathrm{H}$. et al. Age-associated increase in number of CD4+CD8+ intestinal intraepithelial lymphocytes in rats. Eur. J. Immunol. 22, 159-164 (1992).

40. Ohnmacht, $C$. et al. The microbiota regulates type 2 immunity through RORgt+ T cells. Science 349, 989-993 (2015).

41. Sefik, E. et al. Individual intestinal symbionts induce a distinct population of RORgamma(+) regulatory T cells. Science 349, 993-997 (2015).

42. Bain, C. C. et al. Constant replenishment from circulating monocytes maintains the macrophage pool in the intestine of adult mice. Nat. Immunol. 15, 929-937 (2014).

43. Harusato, A. et al. Early-life microbiota exposure restricts myeloid-derived suppressor cell-driven colonic tumorigenesis. Cancer Immunol. Res. 7, 544-551 (2019).

44. Constantinides, M. G. et al. MAIT cells are imprinted by the microbiota in early life and promote tissue repair. Science 366, eaax6624 (2019).

45. Legoux, F. et al. Microbial metabolites control the thymic development of mucosal-associated invariant T cells. Science 366, 494-499 (2019).

46. Al Nabhani, Z. et al. Excess calorie intake early in life increases susceptibility to collitis in the adult. Nat. Metabol. 1, 1101-1109 (2019).

47. Knoop, K. A., McDonald, K. G., McCrate, S., McDole, J. R. \& Newberry, R. D. Microbial sensing by goblet cells controls immune surveillance of luminal antigens in the colon. Mucosal Immunol. 8, 198-210 (2015).

48. Netea, M. G. et al. Trained immunity: A program of innate immune memory in health and disease. Science 352, aaf1098 (2016).

49. Natoli, G. \& Ostuni, R. Adaptation and memory in immune responses. Nat. Immunol. 20, 783-792 (2019)

50. Netea, M. G., Schlitzer, A., Placek, K., Joosten, L. A. B. \& Schultze, J. L. Innate and adaptive immune memory: an evolutionary continuum in the host's response to pathogens. Cell Host Microbe 25, 13-26 (2019).

51. Pradeu, T. \& Du Pasquier, L. Immunological memory: What's in a name? Immunol. Rev. 283, 7-20 (2018).

52. Lorenz, K. Studies in Animal and Human Behaviour. (Harvard University Press, 1970).

53. Hozumi, N. \& Tonegawa, S. Evidence for somatic rearrangement of immunoglobulin genes coding for variable and constant regions. Proc. Natl. Acad. Sci. USA 73, 3628-3632 (1976).

54. Kandel, E. R., Dudai, Y. \& Mayford, M. R. The molecular and systems biology of memory. Cell 157, 163-186 (2014).

55. Cantone, I. \& Fisher, A. G. Epigenetic programming and reprogramming during development. Nat. Struct. Mol. Biol. 20, 282-289 (2013).

56. El Aidy, S. et al. Temporal and spatial interplay of microbiota and intestinal mucosa drive establishment of immune homeostasis in conventionalized mice. Mucosal Immunol. 5, 567-579 (2012).

57. Melum, E. et al. Control of CD1d-restricted antigen presentation and inflammation by sphingomyelin. Nat. Immunol. 12, 1644-1655 (2019).

58. Scheer, S. et al. Early-life antibiotic treatment enhances the pathogenicity of CD4 (+) T cells during intestinal inflammation. J. Leukoc. Biol. 101, 893-900 (2017).

59. Lynn, M. A. et al. Early-life antibiotic-driven dysbiosis leads to dysregulated vaccine immune responses in mice. Cell Host Microbe 23, 653-660 (2018). e655.

60. Clarke, T. B. et al. Recognition of peptidoglycan from the microbiota by Nod1 enhances systemic innate immunity. Nat. Med. 16, 228-231 (2010).
61. Deshmukh, H. S. et al. The microbiota regulates neutrophil homeostasis and host resistance to Escherichia coli K1 sepsis in neonatal mice. Nat. Med. 20, 524-530 (2014)

62. Trompette, A. et al. Gut microbiota metabolism of dietary fiber influences allergic airway disease and hematopoiesis. Nat. Med. 20, 159-166 (2014).

63. Luo, Y. et al. Microbiota from obese mice regulate hematopoietic stem cell differentiation by altering the bone niche. Cell Metab. 22, 886-894 (2015).

64. Khosravi, A. et al. Gut microbiota promote hematopoiesis to control bacterial infection. Cell Host Microbe 15, 374-381 (2014).

65. Silva, S. V., Garcia-Souza, E. P., Moura, A. S. \& Barja-Fidalgo, C. Maternal protein restriction during early lactation induces changes on neutrophil activation and TNF-alpha production of adult offspring. Inflammation 33, 65-75 (2010).

66. Miyazoe, I., Taniguchi, M. \& Takemori, T. The analysis of immature lymphoid precursors stored in longterm bone marrow culture. Microbiol Immunol. 32, 607-620 (1988).

67. Chassin, C. et al. miR-146a mediates protective innate immune tolerance in the neonate intestine. Cell Host Microbe 8, 358-368 (2010).

68. Schuijs, M. J. et al. Farm dust and endotoxin protect against allergy through A20 induction in lung epithelial cells. Science 349, 1106-1110 (2015).

69. Oshima, N. et al. A20 is an early responding negative regulator of Toll-like receptor 5 signalling in intestinal epithelial cells during inflammation. Clin. Exp. Immunol. 159, 185-198 (2010).

70. Naik, S. et al. Inflammatory memory sensitizes skin epithelial stem cells to tissue damage. Nature 550, 475-480 (2017).

71. Pezoldt, J. et al. Neonatally imprinted stromal cell subsets induce tolerogenic dendritic cells in mesenteric lymph nodes. Nat. Commun. 9, 3903 (2018).

72. Mucida, D. et al. Reciprocal TH17 and regulatory $\mathrm{T}$ cell differentiation mediated by retinoic acid. Science 317, 256-260 (2007).

73. Prioult, G. \& Nagler-Anderson, C. Mucosal immunity and allergic responses: lack of regulation and/or lack of microbial stimulation? Immunol. Rev. 206, 204-218 (2005).

74. Ubeda, C. et al. Vancomycin-resistant Enterococcus domination of intestinal microbiota is enabled by antibiotic treatment in mice and precedes bloodstream invasion in humans. J. Clin. Invest. 120, 4332-4341 (2010).

75. Subramanian, S. et al. Persistent gut microbiota immaturity in malnourished Bangladeshi children. Nature 510, 417-421 (2014).

76. Fulde, M. et al. Neonatal selection by Toll-like receptor 5 influences long-term gut microbiota composition. Nature 560, 489-493 (2018).

77. Goethel, A. et al. Nod2 influences microbial resilience and susceptibility to colitis following antibiotic exposure. Mucosal Immunol. 12, 720-732 (2019).

78. $\mathrm{Wu}, \mathrm{H}$. J. et al. Gut-residing segmented filamentous bacteria drive autoimmune arthritis via T helper 17 cells. Immunity 32, 815-827 (2010).

79. Eberl, G. Immunity by equilibrium. Nat. Rev. Immunol. 16, 524-532 (2016).

80. Osborne, L. C. et al. Coinfection. Virus-helminth coinfection reveals a microbiotaindependent mechanism of immunomodulation. Science 345, 578-582 (2014).

81. Baldridge, M. T. et al. Commensal microbes and interferon-lambda determine persistence of enteric murine norovirus infection. Science 347, 266-269 (2015).

82. Pronovost, G. N. \& Hsiao, E. Y. Perinatal Interactions between the Microbiome, Immunity, and Neurodevelopment. Immunity 50, 18-36 (2019).

83. Kuhl, P. K. Brain mechanisms in early language acquisition. Neuron $67,713-727$ (2010).

84. Benes, F. M. Konrad Lorenz, 1903-1989. Am. J. Psychiatry 161, 1767 (2004).

85. Cryan, J. F. \& Dinan, T. G. Mind-altering microorganisms: the impact of the gut microbiota on brain and behaviour. Nat. Rev. Neurosci. 13, 701-712 (2012).

86. Chu, C. et al. The microbiota regulate neuronal function and fear extinction learning. Nature 574, 543-548 (2019).

87. Yan, F. et al. Neonatal colonization of mice with LGG promotes intestinal development and decreases susceptibility to colitis in adulthood. Mucosal Immunol. 10, 117-127 (2017). 